\title{
The Evidence of Invalidation of the BH model at the GC and the Existence of Magnetic Monopoles
}

\author{
Qiu-He Peng ${ }^{1, a}$ \\ ${ }^{1}$ Department of Astronomy, Nanjing University, Nanjing, 210093, China
}

\begin{abstract}
.
\end{abstract}
\section{Discovery of strong radial magnetic field and its effect}

New progress of radio astronomical observations near the GC was reported two years ago. The measurement of an abnormally strong radial magnetic field near the GC was reported by Eatough et al. (2013). The very important result is as follows, at $r=0.12 \mathrm{pc}$ in particular, the lower limit of the outward radial magnetic field near the GC is:

$$
B \geq 8\left[\frac{R M}{66.960 \mathrm{~m}^{-2}}\right]\left[\frac{n_{e}}{26 \mathrm{~cm}^{-3}}\right]^{-1} \mathrm{mG} .
$$

It is well known that the interstellar magnetic field in the Galaxy is usually along the Galactic spiral arms, and the average strength of the magnetic field is about $1 \mu$ Gauss. So the magnetic field shown in eq. (1) is abnormally strong.

Plasma material of the accretion disk in the vicinity of the GC is prevented from approaching to the GC by the strong radial magnetic field, due to the magnetic freeze effect, when the kinematic energy density of the material is less than the energy density of the magnetic field, or the magnetic field is stronger than the Alphen critical value :

$$
B>B_{\text {Alfen }}=\left(4 \pi \rho v_{\text {rot }}^{2}\right)^{1 / 2},
$$

Where is the rotation velocity of the accretion disk around the GC and $\rho$ is the mass density. Making use of the relation $\frac{v_{\text {rot }}}{c}=\sqrt{\frac{R_{S}}{r}}$, we then have

$$
B_{\text {Alfen }}=\left[4 \pi \rho v_{r o t}^{2}\right]^{1 / 2}=\left\{\frac{4 \pi n c^{2}}{N_{A}}\right\}^{1 / 2}\left(\frac{R_{S}}{r}\right)^{1 / 2} \sim 13\left(\frac{n}{10^{4} \mathrm{~cm}^{-3}}\right)^{1 / 2}\left(\frac{R_{S}}{r}\right)^{1 / 2} \text { Gauss, }
$$

where $R_{S}$ is the Schwarzschild radius, and the mass of the central massive object is $4.6 \times 10^{6} \mathrm{~m}_{\odot}$. at $r \sim 0.12 \mathrm{pc},\left(\frac{R_{S}}{r}\right)^{1 / 2}=2 \times 10^{-3}$, thus,

$$
B_{\text {Alfen }} \sim 1.3\left(\frac{n_{e}}{26 \mathrm{~cm}^{-3}}\right)^{1 / 2} \mathrm{mG} .
$$

\footnotetext{
ae-mail: qhpeng@nju.edu.cn
} 
However, the lower limit of the outward radial magnetic field at $r=0.12 \mathrm{pc}$, from the GC is 8 $\mathrm{mG}$.

Some radiations have been detected in the region near the central region of the GC ( see the review article by Falcke and Marko (2013).

a) Some radiations from the radio to the sub-mm wavelength band have been detected in the region around the central black hole with mass $4.3 \times 10^{6} m_{\odot}$ for the GC.

b) $\mathrm{Sgr}^{*}$ is identified as a surprisingly weak X-ray source by Chandra and it is inferred as radiated from the region $\leq 10 R_{g}$ due to the hour long timescale for some detected weak X-ray flare and small NIR flare.

c) The radio flux density shows a flat-to-inverted spectrum, i.e., it rises slowly with frequency with the power peaking around $10^{12} \mathrm{~Hz}$ in the sub $\mathrm{mm}$ band.

As is well known in the popular black hole model of the GC, the radiation from the vicinity of the black hole originates from the inflowing material of the accretion disk (Yuan and Narayan, 2014). But the accretion plasma is clearly prevented from approaching to the GC by the radial magnetic field in the region near $r \sim 0.12 \mathrm{pc}$ around the GC at least as explicitly demonstrated above. Thus, the accretion material cant reach the region near the central b̈lack hole: Consequently, the radiations observed near the GC cannot be emitted by the gas of the accretion disk. This is a dilemma of the standard accretion disk model of black hole at the GC.

\section{The $\alpha$-turbulence dynamo mechanism}

Another important question is how to generate the strong magnetic field near the GC. The most effective mechanism known up to date is the -turbulence dynamo theory proposed by Parker in 1953 (Mestel, 1999, and Charbonneau, 2010) in the solar convection zone. The key idea of the -turbulence dynamo mechanism is that the induced electro-dynamic potential of turbulence is parallel to the magnetic field (Mestel, 1999),

$$
\begin{gathered}
\vec{\epsilon}=\alpha \vec{B} . \\
\alpha \equiv \alpha\left(\sigma t_{c}, \overrightarrow{\vec{v} \cdot \vec{\omega}}\right)=-\frac{\sigma t_{c}}{3 c} \overrightarrow{\vec{v} \cdot \nabla \times \vec{v}}=-\frac{\sigma t_{c}}{3 c} \overrightarrow{\vec{v} \cdot \vec{\omega}}
\end{gathered}
$$

where $\vec{\omega}=\nabla \times \vec{v}$ is the curl of the turbulent velocity of the fluid, and it is approximately equivalent to the large-scale vortex rotational angular velocity. $\sigma$ is the electrical conductivity of the fluid and $t_{c}$ is the typical timescale of the turbulence. Eq.(5) shows that the electro-dynamic potential of turbulence is proportional to the scalar product of the turbulent velocity with vorticity.

In particular, we note that the helical turbulent motions can twist the toroidal field lines to produce the poloidal field as mentioned before. It is the $\alpha$-coefficient which encapsulates this effect of helical motions in the mathematical theory.

Therefore, the principle of the $\alpha$-turbulent dynamo may be briefly sketched as follows: (Priest, 1984) "A toroidal magnetic field $\Rightarrow$ A toroidal electro-dynamic potential $\Rightarrow$ A toroidal current $\Rightarrow A$ poloidal magnetic field $\Rightarrow$ A poloidal electro dynamic potential $\Rightarrow$ A poloidal current $\Rightarrow$ A toroidal magnetic field."

In the theory of $\alpha$-turbulent dynamo, the energy of magnetic field per unit volume is equal to the energy of induced electric current per unit volume, thus, 


$$
\frac{B^{2}}{8 \pi}=n e \epsilon=y n e \alpha\left(\sigma t_{c}, \overrightarrow{\vec{v} \cdot \vec{\omega}}\right) B
$$

or

$$
B=8 \pi e y \alpha\left(\sigma t_{c}, \overrightarrow{\vec{v} \cdot \vec{\omega}}\right) \cdot n,
$$

where $\mathrm{n}$ is the number density of the plasma particles, $\mathrm{y}$ is the degree of ionization. Some relevant data in the Sun are: 1) The mass density in the solar convection zone where dynamo mechanism is valid is $\rho \approx 8 \mathrm{~g} / \mathrm{cm}^{3}$, or the number density of particles is: $n=5 \times 10^{24} \mathrm{~cm}^{3}$; 2) The maximum magnetic field in the solar convection zone is $B_{\max } \sim 10^{5}$ Gauss.

Assuming the validity of the $\alpha$-turbulence dynamo mechanism similar to that in the solar convection zone, and comparing the interstellar magnetic field strength with that of the sunspot, we may estimate the uncertainty of the value of $\alpha$ in terms of the recent observation for the collapsing core W51 e 2 of the star forming region (Koch et al.2012).

We deduce from eq.(8),

$$
\begin{gathered}
B=B_{\odot, \max } \frac{n}{n_{\odot}} \frac{y}{y_{\odot}} \frac{\alpha\left(\sigma t_{c}, \overrightarrow{\vec{v} \cdot \vec{\omega}}\right)}{\alpha\left(\sigma t_{c}, \overrightarrow{\vec{v} \cdot \vec{\omega}}\right)_{\odot}}, \\
B \sim 10^{-19} \cdot \frac{n}{5 \mathrm{~cm}^{-3}} \cdot r\left\{\vec{v}_{t u r b}, \sigma t_{c}\right\} \text { Gauss, }
\end{gathered}
$$

where

$$
r\left\{\vec{v}_{t u r b}, \sigma t_{c}\right\} \text { Gauss }=\frac{y}{y_{\odot}} \frac{\alpha\left(\sigma t_{c}, \overrightarrow{\vec{v} \cdot \vec{\omega}}\right)}{\alpha\left(\sigma t_{c}, \overrightarrow{\vec{v} \cdot \vec{\omega}}\right)_{\odot}}
$$

Though the turbulent velocity in an interstellar cloud may reach $\vec{v}_{\text {turb }} \sim 10 \mathrm{~km} / \mathrm{s}$, the curl of the turbulence velocity in the interstellar cloud is far smaller than that in the Sun, but both the typical timescale of turbulence, $t_{c}$ and the electric conductivity $(\sigma)$ may be much larger than those of the Sun. Thus, the value of the factor $\alpha\left(\sigma t_{c}, \overrightarrow{\vec{v} \cdot \vec{\omega}}\right)$ is rather uncertain.

However, we may estimate it as follows. In some interstellar cloud, the strongest magnetic field may reach $20 \mathrm{mG}$ and the number density is $n=2.7 \times 10^{7} \mathrm{~cm}^{-3}$ near the collapsing core W51 e2 (Koch et al.2012). Using eq. (12), the uncertain factor, $r\left\{\vec{v}_{t u r b}, \sigma t_{c}\right\}$, may be determined in terms of the observed data for W51 e2 just delineated, thus

$$
r\left\{\vec{v}_{t u r b}, \sigma t_{c}\right\} \sim 3.7 \times 10^{10}
$$

In recent work by Qiu et al. (2014), Submillimeter Array Observations of Magnetic Fields in a $\mathrm{H}_{2}$ molecular cloud has been made. the magnetic field is estimated about $1 \mathrm{mG}$, and the corresponding number density is about $2.7 \times 10^{5} \mathrm{~cm}^{-3}$. Thus

$$
r\left\{\vec{v}_{\text {turb }}, \sigma t_{c}\right\} \sim 1.85 \times 10^{11} .
$$

The difference of (13) with (12) is a factor of 5 and it is in the uncertainty region of the $\alpha$-coefficient (it may be reach to (1-2) orders of magnitude, see Charbonneau 2010).

Using Eq.(12) in Eq.(10), with the observed electron number density $n \sim 26 \mathrm{~cm}^{-3}$ at the distance of $0.12 \mathrm{pc}$ from the Galactic Center (Eatough et al., 2013), the resulting magnetic field is given by 


\section{EPJ Web of Conferences}

$B \leq 0.1 \mu \mathrm{G}$ which is five orders of magnitude smaller than the observed lower limit for the field strength, $8 \mathrm{mG}$ (Eatough et al., 2013).

At this point, we would like to mention that such strong magnetic field at the distance of $0.12 \mathrm{pc}$ from the GC cannot be generated by the recent Magnetically arrested accretion disk model (Yuan and Narayan, 2014), although a strong vertical bipolar magnetic field is pushed into the central black hole by the thermal and ram pressure of the accreting gas and the maximum magnetic field strength at the horizon $\left(R_{S} \approx 10^{12} \mathrm{~cm}\right)$ is roughly $10^{3}$ Gauss.

\section{Our Model of supermassive object with magnetic monopoles (SMOMM)}

We note that the important discovery of very strong radial magnetic field in the vicinity of the GC is consistent with the prediction from our model of supermassive object (SMO) with magnetic monopoles (Peng and Chou 2001). Thus, it is plausible to believe that this is just the astronomical evidence needed for the existence of magnetic monopoles as predicted by the grand unified theory of particle physics. In addition, the observed radiation from radio to sub-mm wavelength band with power peaking around $10^{12} \mathrm{~Hz}$ in the sub-mm band and the $\mathrm{x}$-ray radiation near the GC are also essentially in agreement with the prediction of our model ( Peng and Chou 2001). In other words, the dilemma of the standard accretion disk model with supermassive black holes at the GC could be solved.

We have investigated the (non-black hole) model of supermassive object (SMO) with magnetic monopoles in a series of papers since 1985 (Peng, Li and Wang 1985; Peng, Wang and Li., 1985; Peng, 1989; Peng and Chou , 1998; Peng and Chou, 2001; Peng , 2002 ), and the main ideas of our model are as follows ( see Peng and Chou, 2001):

1) The fact that magnetic monopoles (M) may catalyze nucleons to decay (the Rubakov-Callan [RC] effect, $p M \rightarrow e^{+} \pi^{0} M(85 \%)$ or $p M \rightarrow e^{+} \mu^{+} \mu^{-} M(15 \%)$, with the number of baryons being nonconserved) as predicted by the grand unified theory of particle physics is invoked as the main energy source of quasars and active galactic nuclei (AGN). The supermassive central black hole in the standard model is replaced by a supermassive object containing magnetic monopoles. And the accretion disk acts only as a minor energy supply.

2) The gravitational effect around the SMOMM in the Galactic center is similar to that around a massive black hole. However, the supermassive Object containing sufficient magnetic monopoles has neither the horizon nor the central singularity. This is because the reaction rate of the nucleon decay catalyzed by magnetic monopoles is proportional to the square of mass density. Both the leptons and photons from the decay are emitted outward, and the central density cannot approach infinity. Combined with the RC effect from particle physics, our model can avoid the central singularity problem in the standard model of black hole theory.

Some predictions about the GC in our model are as follows (Peng and Chou 2001):

1) Plenty of positrons are produced from the direction of the GC, the productionrate is $10^{43} e^{+} / \mathrm{sec}$ or so. This prediction is quantitatively confirmed by a high-energy astrophysical observation ( $(3.4-$ $6.30) \times 10^{42} e^{+} \mathrm{sec}^{-1}$, Knödlseder et al., 2003).

2) Some higher energy radiation above $0.5 \mathrm{MeV}$ may be emitted. The integral energy of the high energy radiation is much higher than both the total energy of the spectra of electron and positron annihilation, and the total thermal luminosity of the central object. This prediction is also consistent with observations. 
3) The magnetic monopole condensed in the core region of the supermassive object can generate radial magnetic field. The magnetic field strength at the surface of the object is about 20-100 Gauss (the radius of the object is about $8.1 \times 10^{15} \mathrm{~cm}$ or $1.1 \times 10^{4} R_{S}\left(R_{S}\right.$ is the Schwarzschild radius).

We declared previously in our article (Peng and Chou 2001) that this prediction is the most crucial one, which can be testified by future radio observations.

Because the decrease of the magnetic field strength is proportional to the inverse square of the distance from the source, so we have $B \approx(10-50) \mathrm{mG}$ at $r=0.12 \mathrm{pc}$.

4) The super-massive objects containing saturated magnetic monopoles in the centers of all the AGNs in the region $D \leq 50 \mathrm{Mpc}$ from the Earth may be the sources of observed ultra-high energy cosmic rays (for details of this topic see Peng(2002)).

5) The surface temperature of the super-massive object in the Galactic center is about $120 \mathrm{~K}$ and the corresponding spectrum peak of the thermal radiation is at $10^{12} \mathrm{~Hz}$ in the sub-mm wavelength regime. This prediction is quantitatively basically consistent with the recent observation (Falcke and Marko, 2013) on the quatitative.

The non- thermal radiation such as synchrotron radiation, may be emitted due to the motion of the relativistic electrons in the magnetic field.

\section{Conclusions}

In conclusion, we have demonstrated that the radiations observed in the contiguous region of the central black hole cannot be emitted by the gas of the disk since the accreting plasma is prevented from approaching to the GC by the ultra strong magnetic fields. In addition, we have also shown that the observed strong radial magnetic fields near the GC by Eatough et al. (2013), cannot be generated by the -turbulence dynamo mechanism of Parker because qualitative estimate gives a magnetic field strength six orders of magnitude smaller than the observed field strength at $r=0.12 \mathrm{pc}$. The dilemma of the standard model for quasars and AGNs can be avoided if the central black hole in the standard model is replaced by a supermassive stellar object containing magnetic monopoles. The radiations emitted from the inner region of the galactic nucleus and the discovery of the strong radial magnetic field near the GC can all be naturally explained by our model (Peng and Chou 2001). Moreover, the observed ultra-strong radial magnetic field in the vicinity of the GC may be considered as important astronomical evidence for the existence of magnetic monopoles as predicted by the Grand Unified Theory of particle physics.

\section{References}

[1] Charbonneau, P. (2013). Dynamo Models of the Solar Cycle. Solar and Stellar Dynamos. Springer Berlin Heidelberg.

[2] Eatough, R. P., Falcke, H., Karuppusamy, R., et al. (2013). A strong magnetic field around the supermassive black hole at the centre of the galaxy.. Nature, 501(7467), 391-394.

[3] Falcke, H., and Markoff, S. (2013). Toward the event horizonthe supermassive black hole in the galactic center. Classical \& Quantum Gravity, 30(24), 6100-6105.

[4] J. Knödlseder, V. Lonjou, P. Jean, et al. (2003). Early spi/integral constraints on the morphology of the $511 \mathrm{kev}$ line emission in the 4th galactic quadrant. Astronomy \& Astrophysics, 411(1), L457-L460. 
[5] Koch, P. M., Tang, Y. W., and Ho, P. T. P. (2012). Magnetic field strength maps for molecular clouds: a new method based on a polarization - intensity gradient relation. Astrophysical Journal, 747(1), 833-833.

[6] Peng, Q. (1989). The critical and the saturation content of magnetic monopoles in rotating relativistic objects. Astrophysics \& Space Science, 154(2), 271-279.

[7] Peng, Q. (2002). The Origin of the ultra-high energy cosmic ray (A model of quasars and AGNs with magnetic monopoles). High Energy Physics and Nuclear Physics, 26 (2002)104 (in Chinese).

[8] Peng Q., Li, Z., and Wang, D. CONTENT OF MAGNETIC MONOPOLES IN QUASARS, GALACTIC NUCLEI AND STARS AND THEIR ASTROPHYSICAL EFFECTS[J]. Science in China Ser A, 1985, 28(09):970-977.

[9] Peng, Q., Wang, D., and Li, Z. (1985). A monopole model for the galactic nuclei. Science Bulletin(8).

[10] Peng, Q. H., and Chou, C. K. (1997). A model of quasars and agns with magnetic monopoles. Astrophysics \& Space Science, 257(1), 149-159.

[11] Peng, Q. H., and Chou, C. K. (2001). High-energy radiation from a model of quasars, active galactic nuclei, and the galactic center with magnetic monopoles. Astrophysical Journal Letters, 551(1).

[12] Priest, E. R. (2000). Solar magnetohydrodynamics. Physics Today, 36(1), 69.

[13] Qiu, K., Zhang, Q., Menten, K. M., et al. (2014). Submillimeter array observations of magnetic fields in g240.31+0.07: an hourglass in a massive cluster-forming core. Astrophysical Journal Letters, 794(1).

[14] Yuan, F., and Narayan, R. (2014). Hot accretion flows around black holes. Annual Review of Astronomy \& Astrophysics, 52(1), 529-588.

[15] Zamaninasab, M., Clausen-Brown, E., Savolainen, T., et al. (2014). Dynamically important magnetic fields near accreting supermassive black holes.. Nature, 510(7503), 126-128. 